\title{
Dendritic cells from aged subjects contribute to chronic airway inflammation by activating bronchial epithelial cells under steady state
}

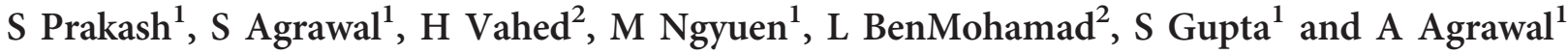

The mechanisms underlying the increased susceptibility of the elderly to respiratory infections are not well understood. The crosstalk between the dendritic cells (DCs) and epithelial cells is essential in maintaining tolerance as well as in generating immunity in the respiratory mucosa. DCs from aged subjects display an enhanced basal level of activation, which can affect the function of epithelial cells. Our results suggest that this is indeed the scenario as exposure of primary bronchial epithelial cells (PBECs) to supernatants from unstimulated DCs of aged subjects resulted in activation of PBECs. The expression of CCL20, CCL26, CXCL10, mucin, and CD54 was significantly increased in the PBECs exposed to aged DC supernatants, but not to young DC supernatants. Furthermore, aged DC supernatants also enhanced the permeability of the PBEC barrier. We also found that DCs from aged subjects spontaneously secreted increased levels of pro-inflammatory mediators, interleukin-6, tumor necrosis factor (TNF)- $\alpha$, and metalloproteinase A disintegrin family of metalloproteinase 10 , which can affect the functions of PBECs. Finally, we demonstrated that TNF- $\alpha$, present in the supernatant of DCs from aged subjects, was the primary pro-inflammatory mediator that affected PBEC functions. Thus, age-associated alterations in DC-epithelial interactions contribute to chronic airway inflammation in the elderly, increasing their susceptibility to respiratory diseases.

\section{INTRODUCTION}

The incidence of respiratory diseases including asthma and chronic obstructive pulmonary disease increases significantly with advancing age. ${ }^{1-6}$ This, coupled with increase susceptibility to respiratory infections, is the major cause of morbidity and mortality in the elderly. Respiratory diseases in the aged population are also often severe and frequently require hospitalization. ${ }^{7,8}$ Therefore, there is an urgent need to identify the underlying mechanisms.

Within the lung, airway epithelial cells (AECs) and dendritic cells (DCs) are co-localized in close proximity, and are the first group of cells to encounter inhaled pathogens, allergens, and environmental pollutants. ${ }^{9-11}$ AECs and DCs interact with each other through the release of cytokines and other soluble mediators and through direct cell-cell contact. These interactions are considered to have an important role in responding to infections in the lung. For example, Hammad et al. ${ }^{10}$ demonstrated that the migration of lung DC to the mediastinal nodes in response to lipopolysaccharide inhalation was largely dependent on Toll like receptor 4 (TLR4) signaling on epithelial cells. Furthermore, activation of AECs by viruses or bacteria induces the production of pro-inflammatory cytokines and type I and III interferons (IFNs), which activate DCs. ${ }^{9}$ In addition to responding to infections, another major role of DCAEC crosstalk is to maintain immune homeostasis or tolerance at the mucosal surfaces. ${ }^{10}$ Thus, alteration in the functions of both these cells can result in the loss of tolerance and chronic inflammation of the airways.

Advancing age significantly impacts DC functions. ${ }^{12}$ These include their capacity to phagocytose antigens, ${ }^{13}$ migration to lymph nodes, ${ }^{13}$ response to pathogens, ${ }^{14,15}$ and impaired secretion of certain key cytokines such as IFN- $\alpha$ and IFN- $\lambda .^{14-16}$ Furthermore, DCs from the elderly display increased basal level of activation as evidenced by increased activation of nuclear factor $\kappa \mathrm{B},{ }^{17}$ which has a major role in the secretion of proinflammatory mediators by DCs. Increased basal level nuclear

${ }^{1}$ Division of Basic and Clinical Immunology, Department of Medicine, University of California-Irvine, Irvine, California, USA and ${ }^{2}$ Cellular and Molecular Immunology Laboratory, Gavin Herbert Eye Institute, University of California-Irvine, Irvine, California, USA. Correspondence: A Agrawal (aagrawal@uci.edu) 
factor $\mathrm{\kappa B}$ activation in aged DCs may result in an enhanced secretion of various molecules under steady state, in the absence of infection. We hypothesize that, because of the close proximity of DCs to AECs, continuous exposure of AECs to these secreted molecules may affect their function and increase the risk of respiratory diseases in the elderly. Most studies have focused on the modulation of DC functions by AECs and less is understood about the effect of DCs on AEC functions.

The objective of the present study was to investigate whether the increased basal level activation of aged DCs results in release of soluble mediators that affect the function of bronchial epithelial cells with a view to understand their role in ageassociated increased susceptibility to respiratory diseases.

\section{RESULTS}

Aged DC supernatant activates primary bronchial epithelial cells (PBECs)

The exposure of PBECs to pro-inflammatory cytokines and other factors during an infection leads to the upregulation of certain molecules that aid in clearance of infection. ${ }^{18} \mathrm{It}$ also results in increased permeability to allow infiltration of other cells to the site of infection. As aged DCs display increased basal level of activation, they may secrete proinflammatory mediators without any stimulation, which may activate PBECs. To investigate this, PBECs were cultured with supernatants from unstimulated aged and young DCs and examined for the expression of surface activation markers. Incubation of PBECs with supernatants from unstimulated aged DCs resulted in a significant $(P<0.05)$ upregulation of the adhesion and co-stimulatory molecule, CD54 (ICAM-1) over control untreated PBECs, whereas supernatants from young DCs had no effect (Figure 1a). The results of flow cytometry were further confirmed by realtime PCR (Figure 1b).

As the activation of PBECs also enhances their permeability, we determined the expression of the tight junction protein, E-cadherin, in PBECs following exposure to supernatants from unstimulated aged vs. young DCs. Incubation of PBECs with supernatants from aged DCs resulted in a significant downregulation $(P<0.05)$ of E-cadherin over control PBECs, whereas supernatants from young DCs had no significant effect $(P>0.05$, Figure 1c). The downregulation of tight junction protein is indicative of increased permeability of PBECs. Quantitative PCR (Q-PCR) was also performed for E-cadherin (CDH-1) and two other tight junction proteins, Claudin (CLD1) and Occludin (OCLD-1). There was no change in the gene expression of these proteins (Figure 1d). This is consistent with recent published reports that downregulation of the tight junction proteins may not be accompanied by changes at the gene level. ${ }^{19}$

Activation of PBECs can result in upregulation of major histocompatibility complex-I. Exposure of PBECs to supernatants from unstimulated DCs from aged and young subjects did not upregulate major histocompatibility complex-I (HLA$A$, - B, and -C; Figure 1e). Mucin secretion is also associated with epithelial cell activation and inflammation. Mucin-1 (MUC1) is induced during airway inflammation and has an important role for the resolution of inflammation during respiratory tract infections. ${ }^{20}$ Therefore, the level of expression of MUC-1 was compared in PBECs following exposure to unstimulated DCs supernatants from aged vs. young subjects. The expression MUC-1 was significantly enhanced $(P<0.05)$ in PBECs exposed to supernatants from aged DCs as compared with those exposed to supernatants from young DCs.

It has been suggested that the expression of TLRs on PBECs, particularly TLR-2, may be upregulated in certain infections. ${ }^{21}$ However, we observed no significant difference $(P>0.05)$ in TLR2 expression on PBECs exposed to supernatants from unstimulated DCs from aged and young.

Altogether, these data suggest that unstimulated aged DCs secrete certain factors that can modulate the functions of PBECs.

\section{Supernatants from aged DCs induce chemokine secretion in PBECs}

Activation of PBECs also results in secretion of various chemokines to signal and attract other immune cells to help fight infections. Therefore, we investigated the effect of supernatants from unstimulated DCs from aged and young subjects on the expression of chemokines by PBECs, as determined by Q-PCR. Figure 2a-d shows that the supernatants from aged DCs induced significant $(P<0.05)$ upregulation of CCL20, CCL26, and CXCL10 chemokines as compared with supernatants from young DCs. No significant difference $(P>0.05)$ was observed in CXCL8 expression. Each of these chemokines have an important role in recruitment of inflammatory cells into the airways; CCL20 (MIP-3 $\alpha$ ) attracts monocytes and DCs, and is upregulated in various respiratory diseases. ${ }^{22}$ CCL26 or Eotaxin-3 attracts eosinophils, ${ }^{23}$ and CXCL10 (IP-10) enhances the infiltration of T cells and may be mast cells. ${ }^{24}$ These data suggest that supernatants from aged DCs directly induce the secretion of chemokines from PBECs, which might be responsible for the recruitment of proinflammatory cells into airways of the elderly under steady state.

\section{Permeability of PBECs is increased after culture with aged DC supernatant}

Our data from Figure 1 suggested that the expression of tight junction protein, E-cadherin, was decreased in PBECs incubated with supernatants from aged DCs. E-cadherin has an important role in maintaining the integrity of the epithelial cell layer and decreased expression of this protein is reported to enhance the permeability of the epithelial cell barrier. ${ }^{25}$ Therefore, we used trans-epithelial electrical resistance (TEER) assay and determined whether exposure of PBECs to supernatants from aged DCs affected the permeability of PBECs. Data in Figure 3a demonstrate that the exposure of PBECs to supernatants from aged DCs resulted in significantly decreased $(P<0.05)$ TEER values by day 7 after culture. In contrast, exposure of PBECs to supernatants from young DCs had no significant effect. These data suggest that aged 

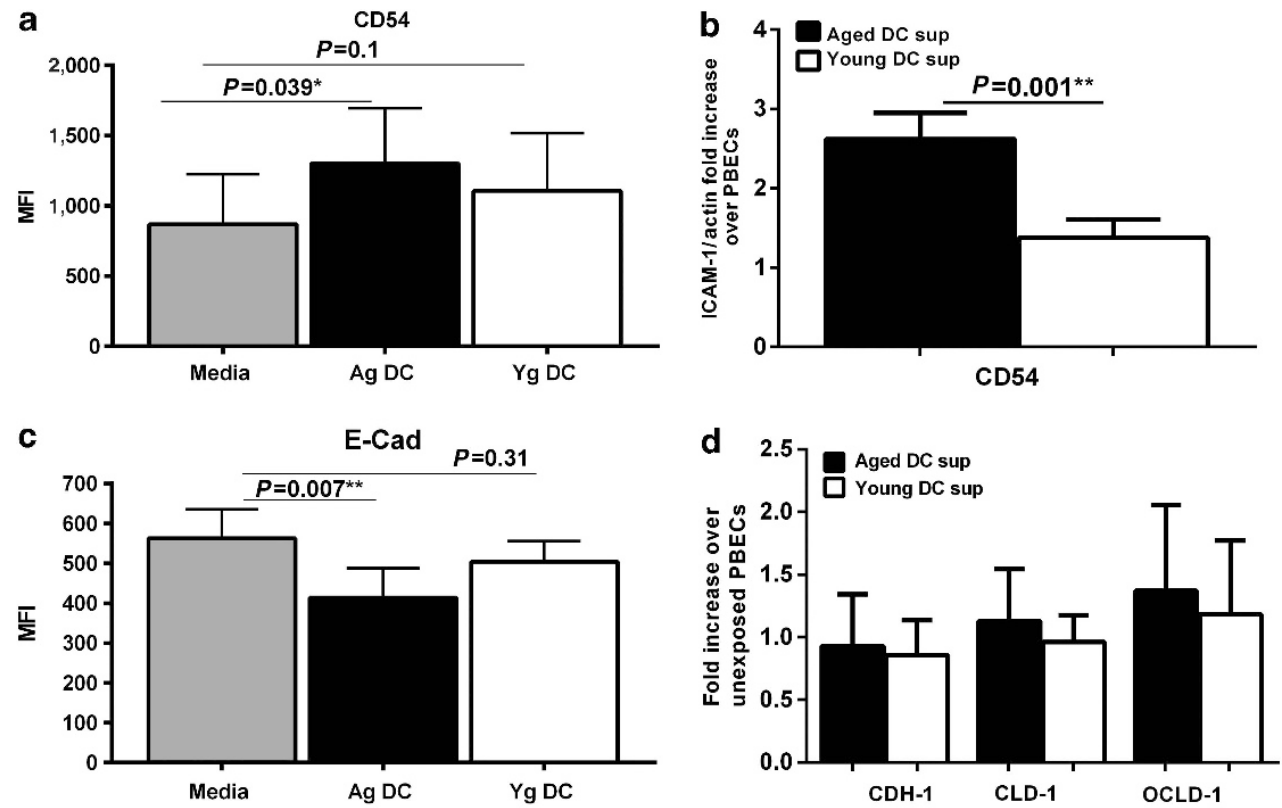

e

HLA-ABC (MHC-I)
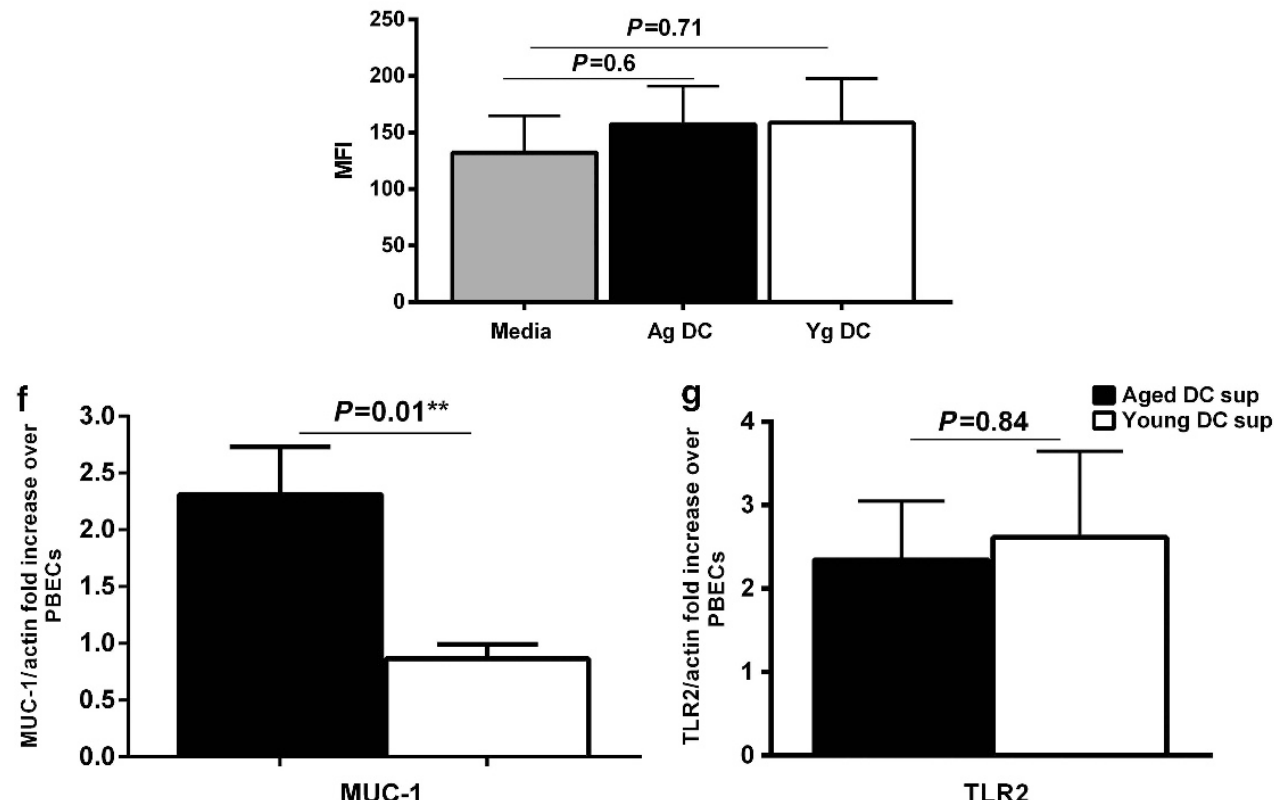

Figure 1 Aged dendritic cell (DC) supernatants activate primary bronchial epithelial cells (PBECs). PBECs were exposed to supernatants (sup) from aged $(\mathrm{Ag})$ or young $(\mathrm{Yg})$ DCs for $6 \mathrm{~h}$ (quantitative PCR (Q-PCR)) or $24 \mathrm{~h}$ (flow cytometry). For Q-PCR, gene expression is normalized with actin and expressed as fold increase over PBECs incubated with media without the DC supernatants. Bar graph depicts the expression of CD54 on PBECs after exposure to aged and young DC supernatants. (a) Mean fluorescence intensity (MFI) as determined by flow cytometry. (b) Gene expression by Q-PCR. Bar graph depicts the expression of adhesion molecules and tight junction proteins on PBECs after exposure to aged and young DC supernatants. (c) MFI as determined by flow cytometry. (d) Gene expression by Q-PCR. (e) Bar graph depicts the MFI of expression of HLA-ABC on PBECs after exposure to aged and young DC supernatants. Gene expression of Mucin-1 (MUC-1) and Toll like receptor 2 (TLR2) in PBECs after exposure to aged and young DC supernatants. (f) MUC-1; (g) TLR2. Data are mean \pm s.e. of six separate aged and young supernatants tested.

DCs spontaneously secrete certain factors that might enhance the permeability of the epithelial cell barrier and allow the infiltration of pro-inflammatory cells in the airways.

To further verify the above results, the passage of dextranfluorescein isothiocyanate (FITC) via the PBEC monolayer was also determined. As shown in Figure $\mathbf{3 b}$, significantly increased $(P<0.05)$ fluorescence was observed in cells incubated with aged DC supernatants as compared with young DC supernatants confirming that supernatants from aged DCs enhanced the permeability of the epithelial cells, by a yet-to-be determined mediator. 

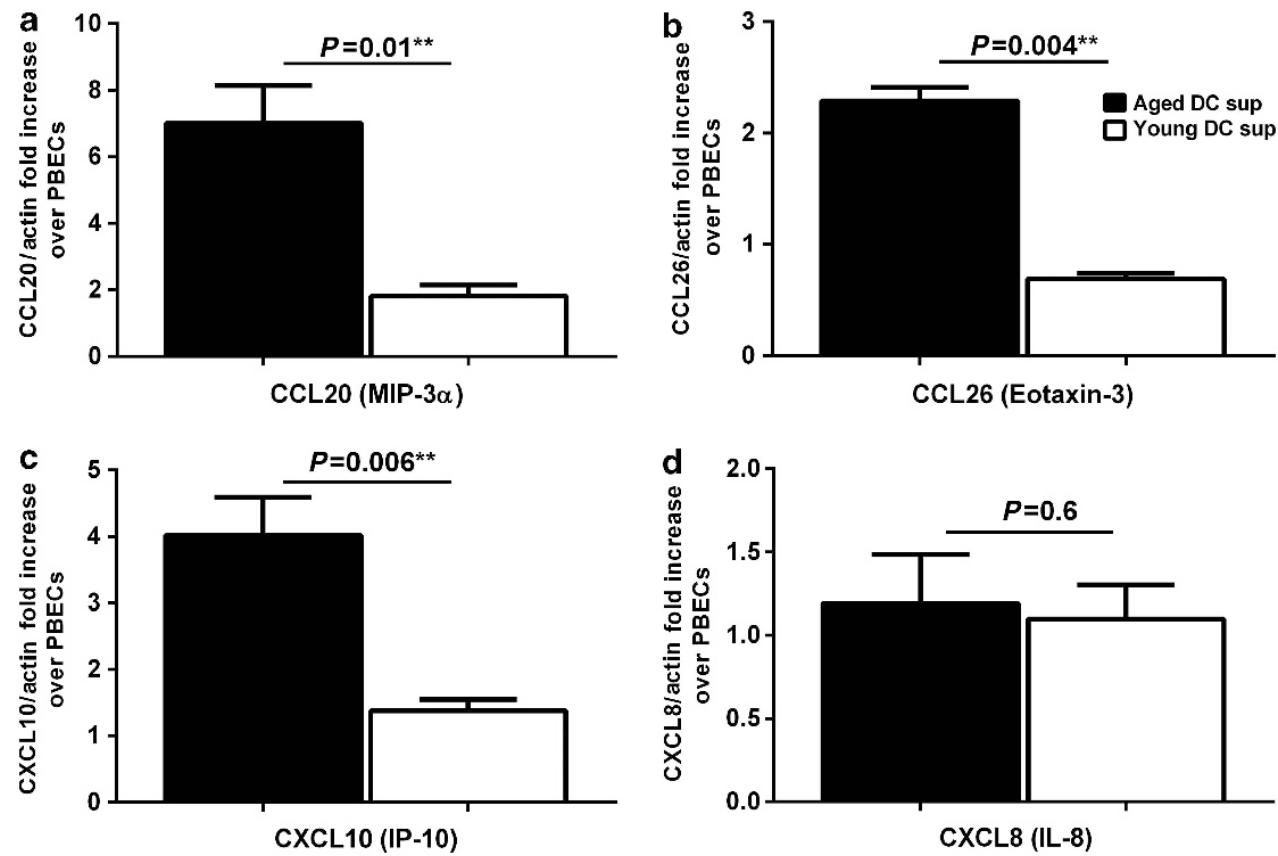

Figure 2 Aged dendritic cell (DC) supernatants induce chemokine secretion in primary bronchial epithelial cells (PBECs). Bar graphs depict the gene expression levels of chemokines in PBECs after exposure to aged and young DC supernatants. Gene expression is normalized with actin and expressed as fold increase over PBECs incubated with media without the DC supernatants. (a) CCL20; (b) CCL26; (c) CXCL10; (d) CXCL8. Data are mean \pm s.e. of six separate aged and young supernatants tested.
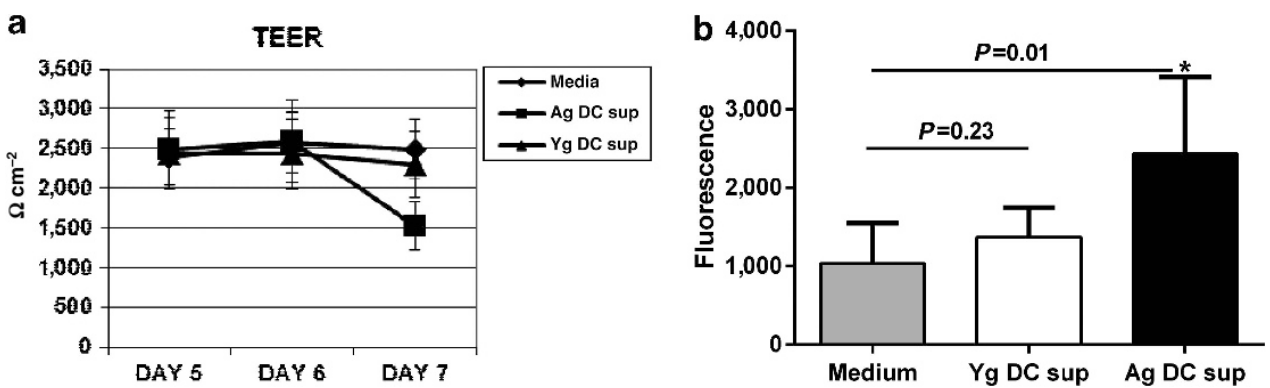

Figure 3 Increased permeability of PBECs after culture with aged dendritic cell (DC) supernatant. primary bronchial epithelial cells (PBECs) were cultured on Transwell till the trans-epithelial electrical resistance (TEER) values reached a steady value. Subsequently, the supernatants (sup) from aged $(\mathrm{Ag})$ and young $(\mathrm{Yg})$ DCs were added and TEER measured. (a) Line graph represents the TEER value of PBECs: before, $24 \mathrm{~h}$, and $48 \mathrm{~h}$ after incubation with DC supernatants. Media alone value is the TEER of PBECs with media alone without the supernatants. (b) Fluorescein isothiocyanate (FITC)-dextran was added $24 \mathrm{~h}$ after the addition of supernatants and fluorescence was measured $24 \mathrm{~h}$ subsequent to the addition. Bar graph represents fluorescent values of FITC-dextran. Data are mean \pm s.e. of three separate aged and young supernatants tested.

Supernatants from aged DCs express enhanced levels of pro-inflammatory mediators and metalloproteinases

To determine possible mediators in supernatants from unstimulated DCs, which might influence PBECs, we analyzed various pro-inflammatory mediators, including cytokines, chemokines, and metalloproteinases. Many (interleukin (IL)-1 $\beta$, IL-12p70, IL-10, IL-23, IFN- $\alpha$ ) were below the detection limit of our assay. Therefore, only those we could detect are displayed in Figure 4a-f. The levels of tumor necrosis factor (TNF)- $\alpha$, IL- 6 , and CXCL10 were significantly greater $(P<0.05)$ in unstimulated supernatants from aged DCs as compared with supernatants from young DCs; the levels of IL$12 \mathrm{p} 40, \mathrm{CXCL} 8$, and CCL2 were comparable between aged and young groups. TNF- $\alpha$ is known to enhance the permeability of the epithelial cell barrier, ${ }^{26}$ whereas IL- 6 is not reported to have a significant effect on the permeability of PBECs by itself but can exert its effect in conjunction with TNF- $\alpha .{ }^{27}$

In addition to cytokines and chemokines, DCs also secrete metalloproteinases, which are known to act on tight junction proteins and enhance the permeability of epithelial cells. Gene array analysis of aged vs. young DCs (unpublished results from our laboratory) suggested that the expression of A disintegrin family of metalloproteinases (ADAM) family members (ADAM10, 12, 19) is increased at the basal level in aged DCs as compared with young DCs. Reports from literature suggest that ADAM family members are involved in the 

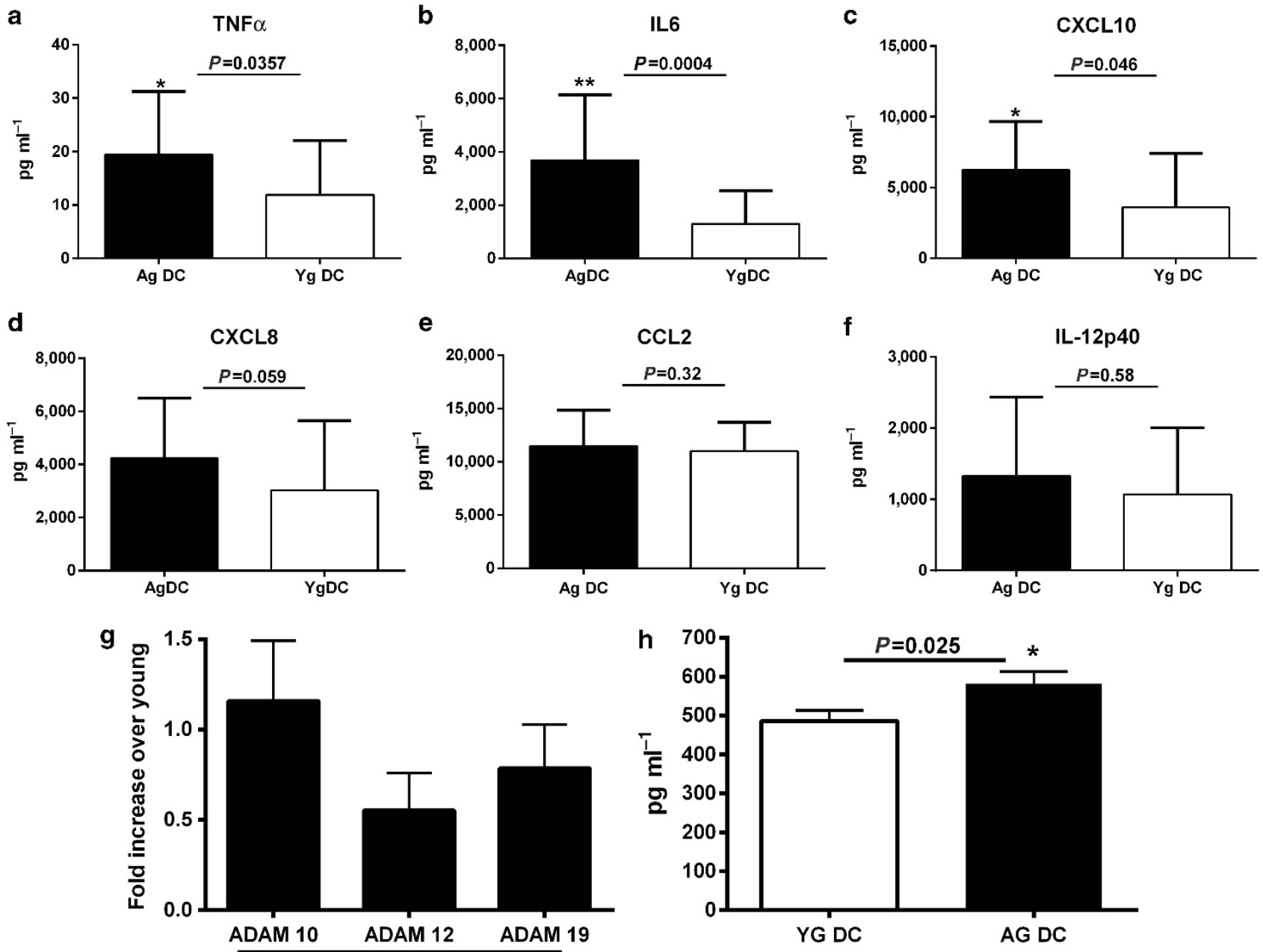

Gene expression in aged DCs

Figure 4 Aged dendritic cell (DC) supernatants express enhanced levels of pro-inflammatory mediators and metalloproteinases. Bar graphs depict the level of cytokines, chemokines, and metalloproteinases present in unstimulated aged $(\mathrm{Ag})$ and young $(\mathrm{Yg}) \mathrm{DC}$ supernatants (sup) as determined by ELISA. (a) Tumor necrosis factor (TNF)- $\alpha$; (b) interleukin (IL)-6; (c) CXCL10; (d) CXCL8; (e) CCL2; (f) IL12p40; (h) A disintegrin family of metalloproteinase 10 (ADAM10). Data are mean \pm s.e. of 25 aged and 25 young subjects. Bar graphs depict the gene expression of metalloproteinases in aged and young DCs; (g) ADAMs. Data are mean \pm s.e. of 12 aged and 12 young subjects.

pathology of several inflammatory diseases. ${ }^{28}$ Therefore, we further confirmed the results by PCR in aged and young DCs. Figure $\mathbf{4 g}$ shows that the expression of ADAM10 was significantly higher $(P<0.05)$ in aged DCs compared with young DCs. ADAM 12 and ADAM19 also displayed an increased expression, although not to the same extent as ADAM10.

As the PBECs were exposed to supernatants from DCs and to further verify our Q-PCR data, we performed ELISA assay for ADAM10 and ADAM12 in supernatants from aged and young DCs. ADAM10 levels were significantly greater $(P<0.05)$ in supernatants from aged DCs as compared with those from young DCs (Figure $\mathbf{4 h}$ ), thus confirming our Q-PCR data. ADAM12 levels were below the detection limits of the assay.

The elderly population enrolled in the study is a stable population, living independently (Table 1). Osteoarthritis was the most common complaint in the aged population.
Table 1 Description of the aged and young cohorts

\begin{tabular}{lcc}
\hline & Young, $\boldsymbol{n}=\mathbf{2 5}$ & Aged, $\boldsymbol{n}=\mathbf{2 5}$ \\
\hline Age (range), years & $(20-32)$ & $(65-93)$ \\
Age (mean), years & 27 & 78 \\
Gender, female & 15 & 19
\end{tabular}

\section{Comorbidities}

Osteoarthritis

$0 \quad 14$

Hypertension

0

8

Dyslipidemia

0

9

\section{Medications}


No significant differences $(P>0.5)$ were found in the secretion of TNF- $\alpha$, IL-6, CXCL-10, and ADAM10 between elderly subjects based on gender, osteoarthritis, hypertension, vitamins, and supplements or dyslipidemia.

Altogether, these data demonstrate that aged DCs secrete spontaneously increased amounts of IL- 6 , TNF- $\alpha$, and ADAMs, which can affect the functions of epithelial cells and enhances the susceptibility of elderly to respiratory infections.

\section{Addition of TNF- $\alpha$ to supernatants from young DCs was sufficient to mimic the effect of aged DC supernatants on PBECs}

Next, we investigated whether addition of TNF- $\alpha$, IL-6, CXCL10 , or ADAM10 mediators to supernatants from young DCs will replicate the activation effects of aged DCs supernatants on epithelial cells. PBECs were incubated with supernatants from young DCs to which one of these mediators were added. As shown in Figure 5a and b, Q-PCR results revealed that addition of TNF- $\alpha$ to young DC supernatants had an effect on PBECs, with almost identical results to aged DC supernatants. The expression of CCL20, CCL26, CXCL10, MUC-1, CD54 was significantly upregulated $(P<0.05)$ in $\mathrm{PBECs}$ exposed to young DCs after addition of TNF- $\alpha$ relative to young DC supernatants without TNF- $\alpha$. No significant effect was observed on the expression of CXCL8, and adhesion molecules, E-cadherin, Occludin, and Claudin (Figure 5c). Similar to aged DC supernatants, TNF- $\alpha$-containing young DC supernatants also reduced the expression of E-cadherin (Figure 5d) as determined by flow cytometry. Addition of a mixture of IL-6, CXCL10, and ADAM10 to TNF- $\alpha$-containing young DC supernatants resulted in almost similar activity as young supernatants containing TNF- $\alpha$ alone. This: (i) indicates that addition of TNF- $\alpha$ inflammatory mediator to young DC supernatants was sufficient to mimic the effect of aged DC supernatants on PBECs; and (ii) suggest that TNF- $\alpha$ in aged supernatants is likely the major inflammatory mediator responsible for the effect on PBEC functions. In support of this result, young DC supernatants containing IL-6, CXCL10, or ADAM10 displayed no significant activity over young DC supernatants without them (Supplementary Figure 1 online).

Altogether, the above results suggest that DCs from aged subjects may contribute to chronic airway inflammation by activating PBECs through a TNF- $\alpha$-mediated mechanism.

\section{DISCUSSION}

The objective of the present study was to investigate the mechanisms underlying the apparent increase in severity of chronic airway inflammation in the elderly that leads to a

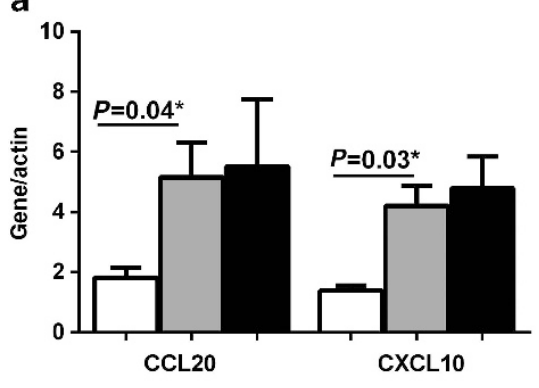

d

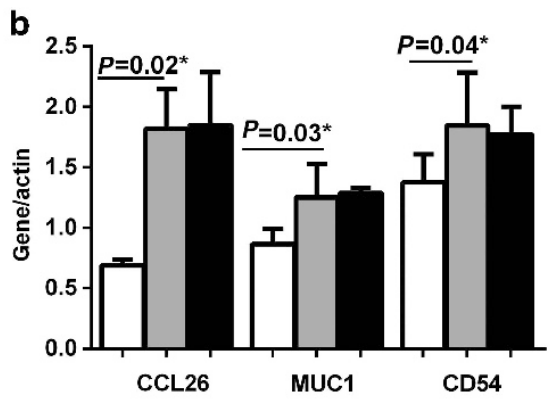

e

CD54

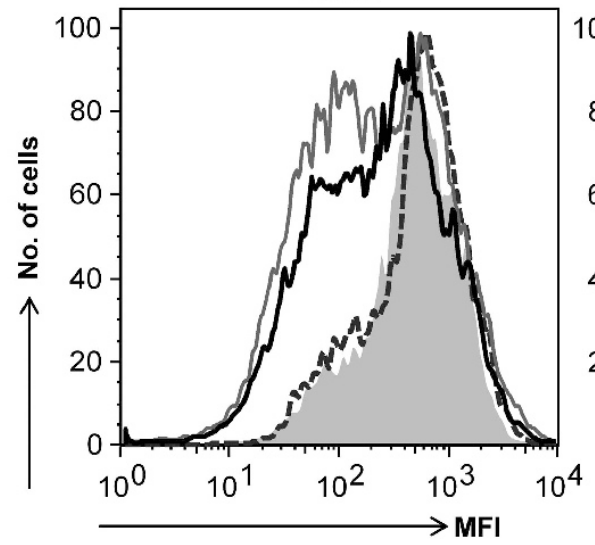

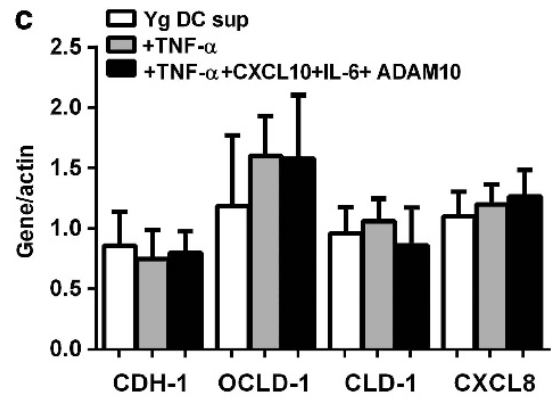

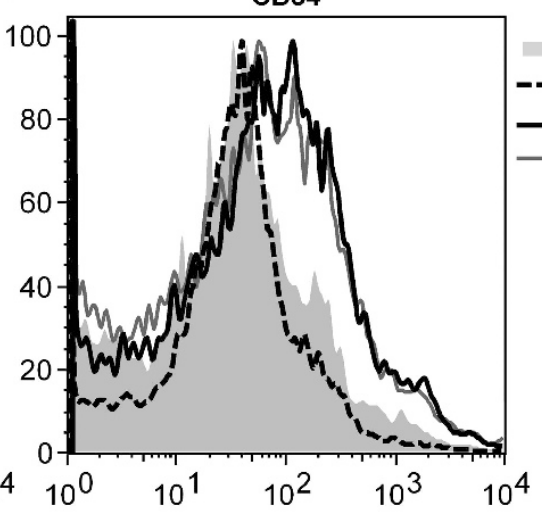

Figure 5 Addition of tumor necrosis factor (TNF)- $\alpha$ to young dendritic cell (DC) supernatants mimics the action of aged DC supernatants on primary bronchial epithelial cells (PBECs). PBECs were incubated with young DC supernatants to which TNF- $\alpha$ alone or a mixture of TNF- $\alpha$, interleukin (IL)-6, CXCL10, A disintegrin family of metalloproteinase 10 (ADAM10) was added. Bar graphs depict the gene expression levels of various molecules in PBECs after exposure to the above young DC supernatants. (a) CCL20, CXCL10; (b) CCL26, Mucin-1 (MUC1), CD54. (c) E-cadherin (CDH)-1, Occludin (OCLD)-1, Claudin (CLD)-1, CXCL8. Histograms represent the expression of (d) E-Cadherin; (e) CD54 as determined by flow cytometry in PBECs exposed to the young DC supernatants described above. Data are mean \pm s.e. of four separate young supernatants tested. 
increased susceptibility to respiratory diseases. Our results indicate that increased basal level of activation of aged DCs leads to spontaneous secretion of pro-inflammatory cytokines and metalloproteinases. These factors lead to the TNF- $\alpha$ mediated activation of PBECs resulting in increased permeability and secretion of chemokines. Increased permeability accompanied by enhanced chemokines may lead to infiltration of pro-inflammatory cells in the inflamed airways. The chronic inflammation and the associated airway remodeling can thus increase the risk of respiratory diseases such as asthma and chronic obstructive pulmonary disease as well as enhance the risk of respiratory infections such as influenza and pneumonia, which are all prevalent in the elderly.

Our results demonstrate that unstimulated aged DC supernatants can enhance the expression of CD54 on PBECs (Figure 1a and b). CD54 is not only a marker of PBEC activation but also serves as a receptor for Haemophilus influenzae, Streptococcus pneumoniae, and Rhinovirus. ${ }^{29}$ These three common pulmonary infections are a major cause of health concern in the elderly. In addition to serving as a receptor for Rhinovirus, CD54 also allows adhesion of neutrophils and lymphocytes to the epithelium enhancing inflammation. ${ }^{30}$ Furthermore, CD54 along with CCL26 (Eotaxin) has an important role in migration and binding of eosinophils. ${ }^{31}$ Eotaxin selectively recruits eosinophils via CCR3 expressed on eosinophils and CD54 is important for eosinophil adhesion via LFA-1, a receptor also found on eosinophils. ${ }^{31}$ Both neutrophil and eosinophil have been demonstrated to be increased in inflamed airways of older asthmatics and have a role in airway obstruction and inflammation. ${ }^{32}$

We also observed increased expression of CCL20 and CXCL10 in PBECs cultured with supernatants from aged DCs (Figure 2a-c). Both these chemokines are involved in inflammation of lung. CCL20 (MIP3- $\beta$ ) is reported to be upregulated in the airways of subjects with obstructive airway diseases. ${ }^{33}$ It binds to CCR- 6 on PBECs and induces the secretion of mucus. ${ }^{33}$ It also acts as a major chemoattractant for DCs. ${ }^{34}$ CXCL10 (IP-10) attracts T cells and IP-10 levels are substantially increased in chronic obstructive pulmonary disease patients. ${ }^{35,36}$ Furthermore, it is reported that eosinophil adhesion to CD54 was significantly enhanced by CXCL10 and therefore it functions as a biomarker for virus-induced asthma. ${ }^{37}$

In contrast to chemokines, the expression of TLR2, CXCL8, and major histocompatibility complex-I on the surface of PBECs was not affected by aged DC supernatants. This suggests that the aged DC supernatants do not activate PBECs to the same level as pathogens where a variety of pro-inflammatory mediators are secreted and affect the expression of numerous molecules. For example, IFN- $\alpha$ or IFN- $\lambda$ secreted in by DCs in response to respiratory syncytial virus can upregulate major histocompatibility complex-I expression. ${ }^{38}$ Similarly, TLR2 is found to be upregulated in response to Mycoplasma pneumoniae. ${ }^{39}$ Because DC-epithelial cells crosstalk is mainly via secreted factors, the present study has focused primarily on the effect of soluble mediators secreted by DCs on PBEC functions. However, we do not exclude that a direct cell-to-cell contact between DC and epithelial cells may also influence the functions of both cells. This possibility is currently being investigated in our laboratory and the finding will be the subject of a future report.

We also found that treatment of PBECs with supernatants from aged DCs also resulted in reduced expression of E-cadherin and enhanced permeability of the epithelial cell barrier. Unstimulated aged DCs secreted increased levels of TNF- $\alpha$ (Figure 4a), which is known to activate PBECs and enhance their permeability. This is in keeping with studies from Panda et al. ${ }^{14}$ who also observed increased TNF- $\alpha$ secretion from aged DCs in the absence of any stimulation. TNF- $\alpha$ is reported to be increased in airways of severe, corticosteroid refractory patients with asthma and implicated in airway pathology. ${ }^{27}$ Besides TNF- $\alpha$, IL-6 levels were also significantly enhanced in aged DC supernatants. IL-6 enhances epithelial cell invasion along with other cytokines. ${ }^{26}$ Furthermore, the expression of adhesion proteins is also regulated by metalloproteinases. Maretzky et al. ${ }^{40}$ have reported that E-cadherin is cleaved specifically by ADAM10. Therefore, increased secretion of ADAM10 by aged DCs may also contribute to the decreased expression of E-cadherin in PBECs incubated with supernatants from aged DCs. ADAMs have been demonstrated to have a role in the shedding of proprotein ectodomains-like membrane-anchored cytokines and growth factors. ${ }^{41}$ Recent studies suggest a role of ADAMs in pathophysiology of lung diseases such asthma, ${ }^{42}$ interstitial lung disease, ${ }^{43}$ eosinophilic pneumonia, ${ }^{42}$ and lung cancer. ${ }^{44}$ Furthermore, $\alpha$-hemolysin, the pore-forming toxin of Staphylococcus aureus binds to ADAM10, and is necessary for the toxin to cause cytotoxicity at low concentrations. ${ }^{45}$ Hospital-acquired methicillin-resistant Staphylococcus aureus-related hospitalizations are more common and a major cause of health concern in older individuals. ${ }^{46}$

Culture of PBECs with TNF- $\alpha$-containing young DCs supernatants confirmed that TNF- $\alpha$ present in the aged supernatants was sufficient and is likely responsible for the observed effects on PBECs (Figure 5a-e). In contrast, IL-6, CXCL10, and ADAM10 had no effect on PBEC functions by themselves (Supplementary Figure 1) and supplement with TNF- $\alpha$ is required to lead to activation effect on PBECs (Figure 5a-c). Recent reports also suggest that TNF- $\alpha$ can induce the expression of various chemokines in PBECs. ${ }^{47-49}$ Exposure of PBECs to TNF- $\alpha$ also enhances the expression of $\mathrm{CD} 54^{50}$ and increases the permeability of PBECs by reducing the expression of E-cadherin. ${ }^{51}$ ADAM10 is known to cleave E-cadherin. ${ }^{40}$ However, recombinant ADAM10 does not display the metalloproteinase activity in vitro against E-cadherin. This could be the reason why ADAM10 had no effect on E-cadherin expression. Future studies are required to address whether PBECs from older donors show the same responses to higher levels of TNF- $\alpha$ from DCs as noted here in PBECs of younger donors.

In summary, our results demonstrate that aged DCs display an activated phenotype, which results in the secretion of proinflammatory cytokines and metalloproteases. As DCs are in close proximity to epithelial cells, continuous secretion of these 
mediators by aged DCs might have a profound effect on the functions of PBECs resulting in increased permeability as well as enhanced expression of CD54 and secretion of chemokines. Furthermore, TNF- $\alpha$ secreted by DCs from aged subjects appears to be the primary pro-inflammatory mediator responsible for the activation of PBECs. This may lead to severe and chronic inflammation and airway remodeling in lungs of the elderly, thus enhancing their susceptibility to pulmonary infections and diseases.

\section{METHODS}

Blood donors. Peripheral blood samples were obtained from healthy aged and young volunteers. The age of young donors were between 20 and 35 years and that of aged between 65 and 90 years. Elderly subjects belong to a middle-class socioeconomic status that live independently and lead an active life, although regular physical exercise was not a requirement for participation in the study. Blood was drawn by an Immunology Allergy Fellow from the division. Before the blood draw, the subjects are asked to fill a questionnaire and any subjects suffering from diseases such as diabetes, heart diseases, malignancy, neurological disorders, chronic inflammatory, or autoimmune disorders or those taking drugs that can affect the immune system are excluded from the study. Subjects having any history of recent hospitalization for any disorder including respiratory infections are also excluded. Younger volunteers were students and staff of the University. They also have been undergoing a detailed medical (clinical, hematological, and biochemical) examination each year. Mean age for young subjects was 27 years, whereas was 78 years for the aged subjects. Table 1 provides the description of the cohorts. This study was approved by the Institutional Review Board of the University of California (Irvine, CA).

Isolation and culture of human monocyte-derived DCs. Monocytederived DCs from aged and young subjects were prepared essentially as described. ${ }^{15}$ Peripheral blood mononuclear cells were separated by Ficoll-Hypaque density gradient centrifugation. Monocytes were purified by positive selection with anti-CD14 microbeads (Stemcell Sep, Vancouver, BC, Canada). The purity of the isolated CD14+ monocytes was $>90 \%$, as determined by flow cytometry. For the induction of DC differentiation, purified CD14 + monocytes were cultured in a humidified atmosphere of $5 \% \mathrm{CO}_{2}$ at $37^{\circ} \mathrm{C}$ in RPMI 1640 supplemented with $10 \%$ fetal bovine serum, $1 \mathrm{~mm}$ glutamine, $100 \mathrm{U} \mathrm{ml}^{-1}$ penicillin, $100 \mu \mathrm{g} \mathrm{ml}^{-1}$ streptomycin, $50 \mathrm{ng} \mathrm{ml}^{-1}$ human rGM-CSF (PeproTech, Rocky Hill, NJ), and $10 \mathrm{ng} \mathrm{ml}^{-1}$ human rIL-4 (PeproTech). Half of the medium was replaced every 2 days and DCs (CD14-HLA-DR + CD11c + cells) were collected after 6 days. The purity of the DC was $>95 \%$.

The collected immature aged and young DCs were then cultured in serum-free AIM V medium for $48 \mathrm{~h}$ and supernatants were collected and stored at $-20^{\circ} \mathrm{C}$ until use.

ELISA. Supernatants collected from DCs were assayed for IL-6, TNF$\alpha$, CXCL-8, CXCL-10, CCL-2, and IL-12p40, IL-1 $\beta$, IL-12p70, IL-10, IL-23, IFN- $\alpha$ by Flow Cytomix assay (eBiosciences, San Diego, CA) as per the manufacturer's protocol. ADAM 10 (Uscn Life Science, Wuhan, China) and ADAM12 (R\&D Systems, Minneapolis, MN) were measured using specific ELISAs.

PBECs. PBECs from normal, healthy young individuals were obtained from Cell Applications (San Diego, CA) and maintained in the medium provided by the manufacturer as per their instructions. PBECs were from two different donors (24 years, Caucasian male and 30 years, Caucasian Female) were used for the study.

Flow cytometry. PBECs were cultured in 24-well plates overnight with supernatants (PBEC and DC-conditioned medium were used at 1:1 proportion) from aged and young DCs. For experiments in Figure 5, recombinant TNF- $\alpha\left(500 \mathrm{pg} \mathrm{ml}^{-1}\right)$ or a combination of TNF- $\alpha$, IL-6 (5 $\left.\mathrm{ng} \mathrm{ml}^{-1}\right)$, CXCL-10 (5 $\mathrm{ng} \mathrm{ml}^{-1}$; PeproTech), ADAM10 $\left(1 \mu \mathrm{g} \mathrm{ml}^{-1}\right.$; R\&D Systems) was added to supernatants from young DCs and incubated overnight. Recombinant TNF- $\alpha$ was not active below $500 \mathrm{pg} \mathrm{ml}^{-1}$. Subsequently, the adherent PBECs were collected by scraping with a Teflon scraper. The expression of CD54, HLA-ABC and E-Cadherin on PBECs was determined with flow cytometry using specific antibodies (BD Bioscience, San Jose, CA).

Gene expression. PBECs were exposed to supernatants from aged and young DCs for $6 \mathrm{~h}$. For experiments in Figure 5, recombinant TNF- $\alpha\left(100 \mathrm{pg} \mathrm{ml}^{-1}\right)$ or a combination of TNF- $\alpha$, IL-6 $\left(5 \mathrm{ng} \mathrm{ml}^{-1}\right)$, CXCL-10 (5 $\mathrm{ng} \mathrm{ml}^{-1}$; PeproTech), ADAM10 (1 $\mu \mathrm{g} \mathrm{ml}^{-1}$; R\&D Systems) was added to supernatants from young DCs for $6 \mathrm{~h}$. RNA was extracted using TRIZOL. Real-time PCR was performed using validated specific primers for CD54, MUC-1, TLR2, chemokines (Real-time primers; LLC, Elkins Park, PA). The data were normalized to $\beta$-actin. Relative quantification method was used for calculations and data are expressed as fold increase over controls.

TEER. TEER is the potential difference across the epithelium, which is a measure of the tightness of the cell-cell contacts within the epithelium, can be measured using a pair of electrodes. ${ }^{52}$ PBECs were plated on $0.4 \mu \mathrm{m}, 6.5 \mathrm{~cm}^{2}$ per well, 24-well plates (Corning, HTS Transwell, Tewksbury, MA) and allowed to grow. Cell confluence was monitored by TEER measurements with the Millicell-ERS system (Millipore, Billerica, MA). The TEER value is an indication of the integrity of the epithelial cell monolayers. ${ }^{52}$ Once TEER value reached a steady state of around $1,200-1,300 \Omega \mathrm{cm}^{-2}$ at days 5-6, supernatants from aged or young DCs were added in the bottom chamber underneath the insert membrane and TEER measurement were performed $24 \mathrm{~h}$ after addition. The mean of three measurements per insert was calculated. The electrical resistance of filters without cells (value around $100 \Omega$ ) was subtracted from all samples, and the resistance values were multiplied with the surface area of the inserts.

Permeability was also assayed with a Flourimeter (Molecular Devices, Downingtown, PA) by measuring the basolateral passage of a 4-kDa FITC-dextran (Sigma-Aldrich, St Louis, MO) following application of FITC-dextran to cells apically and incubating for $24 \mathrm{~h}$ at $37^{\circ} \mathrm{C}$. FITC-dextran was added $24 \mathrm{~h}$ after the addition of supernatants.

Statistical analysis. Statistical analysis was performed using Graph pad prism (GraphPad, San Diego, CA). Differences between unstimulated and stimulated conditions were tested using paired $t$-test. Differences between aged and young subjects' DC supernatants were tested using Mann-Whitney test. A $P$-value of $<0.05$ was considered statistically significant.

SUPPLEMENTARY MATERIAL is linked to the online version of the paper at http://www.nature.com/mi

\section{ACKNOWLEDGMENTS}

We thank Institute of Clinical and Translational Science, UCl, for providing the blood from young subjects.

\section{DISCLOSURE}

The authors declared no conflict of interest.

c) 2014 Society for Mucosal Immunology

\section{REFERENCES}

1. Stupka, E. \& deShazo, R. Asthma in seniors: Part 1. Evidence for underdiagnosis, undertreatment, and increasing morbidity and mortality. Am. J. Med. 122, 6-11 (2009). 
2. Diaz-Guzman, E. \& Mannino, D.M. Airway obstructive diseases in older adults: From detection to treatment. J. Allergy Clin. Immunol. 126, 702-709 (2010).

3. Busse, P.J. \& Mathur, S.K. Age-related changes in immune function: effect on airway inflammation. J. Allergy Clin. Immunol. 126, 690-699 (2010).

4. Reed, C.E. Asthma in the elderly: diagnosis and management. J. Allergy Clin. Immunol. 126, 681-687 (2010).

5. Bellia, V. et al. Asthma in the elderly: mortality rate and associated risk factors for mortality. Chest 132, 1175-1182 (2007).

6. Schildgen, $\mathrm{O}$. The lack of protective immunity against RSV in the elderly. Epidemiol. Infect. 137, 1687-1690 (2009).

7. World Health Organization (WHO). Influenza vaccines. Wkly. Epidemiol. Rec. 80, 279-287 (2005).

8. El-Solh, A.A., Niederman, M.S. \& Drinka, P. Nursing home-acquired pneumonia: a review of risk factors and therapeutic approaches. Curr. Med. Res. Opin. 26, 2707-2714 (2010).

9. Banchereau, J. \& Steinman, R.M. Dendritic cells and the control of immunity. Nature 392, 245-252 (1998).

10. Hammad, H. \& Lambrecht, B.N. Dendritic cells and epithelial cells: linking innate and adaptive immunity in asthma. Nat. Rev. Immunol. 8, 193-204 (2008).

11. Lambrecht, B.N. \& Hammad, H. Lung dendritic cells in respiratory viral infection and asthma: from protection to immunopathology. Annu. Rev. Immunol. 30, 243-270 (2012).

12. Agrawal, A. \& Gupta, S. Impact of aging on dendritic cell functions in humans. Ageing Res. Rev. 10, 336-345 (2011).

13. Agrawal, A., Agrawal, S., Cao, J., Su, H., Osann, K. \& Gupta, S. Altered innate immune functioning of dendritic cells in aging humans: role of PI3Kinase signaling pathway. J. Immunol. 178, 6912-6922 (2007).

14. Panda, A. et al. Age-associated decrease in TLR function in primary human dendritic cells predicts influenza vaccine response. J. Immunol. 184, 2518-2527 (2010).

15. Sridharan, A. et al. Age-associated impaired plasmacytoid dendritic cell functions lead to decreased CD4 and CD8 T cell immunity. AGE 33, 363-376 (2011).

16. Prakash, S., Agrawal, S., Cao, J.N., Gupta, S. \& Agrawal, A. Impaired secretion of interferons by dendritic cells from aged subjects to influenza: role of histone modifications. AGE 35, 1785-1797 (2012).

17. Agrawal, A., Tay, J., Ton, S., Agrawal, S. \& Gupta, S. Increased reactivity of dendritic cells from aged subjects to self-antigen, the human DNA. J. Immunol. 182, 1138-1145 (2009).

18. Schleimer, R.P., Kato, A., Kern, R., Kuperman, D. \& Avila, P.C. Epithelium: at the interface of innate and adaptive immune responses. J. Allergy Clin. Immunol. 120, 1279-1284 (2007).

19. Solanas, G., Cortina, C., Sevillano, M. \& Batlle, E. Cleavage of E-cadherin by ADAM10 mediates epithelial cell sorting downstream of EphB signalling. Nat. Cell Biol. 13, 1100-1107 (2011).

20. Kim, K.C. \& Lillehoj, E.P. MUC1 mucin: a peacemaker in the lung. Am. J. Respir. Cell Mol. Biol. 39, 644-647 (2008).

21. Unger, B.L., Faris, A.N., Ganesan, S., Comstock, A.T., Hershenson, M.B. \& Sajjan, U.S. Rhinovirus attenuates non-typeable Hemophilus influenzaestimulated IL-8 responses via TLR2-dependent degradation of IRAK-1. PLoS Pathog. 8, e1002969 (2012).

22. Ito, T., Carson, W.F., Cavassani, K.A., Connett, J.M. \& Kunkel, S.L. CCR6 as a mediator of immunity in the lung and gut. Exp. Cell Res. 317, 613-619 (2011).

23. Garcia, G., Godot, V. \& Humbert, M. New chemokine targets for asthma therapy. Curr. Allergy Asthma Rep. 5, 155-160 (2005).

24. Bradding, P., Walls, A.F. \& Holgate, S.T. The role of the mast cell in the pathophysiology of asthma. J. Allergy Clin. Immunol. 117, 1277-1284 (2006).

25. Sumitomo, T., Nakata, M., Higashino, M., Terao, Y. \& Kawabata, S. Group a streptococcal cysteine protease cleaves epithelial junctions and contributes to bacterial translocation. J. Biol. Chem. 288, 13317-13324 (2013).

26. Coyne, C.B., Vanhook, M.K., Gambling, T.M., Carson, J.L., Boucher, R.C. \& Johnson, L.G. Regulation of airway tight junctions by proinflammatory cytokines. Mol. Biol. Cell 13, 3218-3234 (2002).

27. Hardyman, M.A. et al. TNF- $\alpha$-mediated bronchial barrier disruption and regulation by src-family kinase activation. J. Allergy Clin. Immunol. 132, 665-675.e8 (2013).
28. Crawford, H.C., Dempsey, P.J., Brown, G., Adam, L. \& Moss, M.L. ADAM10 as a therapeutic target for cancer and inflammation. Curr. Pharm. Des. 15, 2288-2299 (2009).

29. Min, J.Y. \& Jang, Y.J. Macrolide therapy in respiratory viral infections. Mediators Inflamm. 2012, 649570 (2012).

30. Wang, S.Z. \& Forsyth, K.D. The interaction of neutrophils with respiratory epithelial cells in viral infection. Respirology 5, 1-9 (2000).

31. Jacoby, D.B., Costello, R.M. \& Fryer, A.D. Eosinophil recruitment to the airway nerves. J. Allergy Clin. Immunol. 107, 211-218 (2001).

32. Porsbjerg, C.M. et al. Relationship between airway pathophysiology and airway inflammation in older asthmatics. Respirology 18, 1128-1134 (2013).

33. Kim, S., Lewis, C. \& Nadel, J.A. CCL20/CCR6 feedback exaggerates epidermal growth factor receptor-dependent MUC5AC mucin production in human airway epithelial (NCl-H292) cells. J. Immunol. 186, 3392-3400 (2011).

34. Haczku, A. The dendritic cell niche in chronic obstructive pulmonary disease. Respir. Res. 13, 80 (2012).

35. Spurrell, J.C., Wiehler, S., Zaheer, R.S., Sanders, S.P. \& Proud, D. Human airway epithelial cells produce IP-10 (CXCL10) in vitro and in vivo upon rhinovirus infection. Am. J. Physiol. Lung Cell Mol. Physiol. 289, 85-95 (2005).

36. Wark, P.A. et al. IFN-gamma-induced protein 10 is a novel biomarker of rhinovirus-induced asthma exacerbations. J. Allergy Clin. Immunol. 120, 586-593 (2007).

37. Davies, J.M. et al. Budesonide and formoterol reduce early innate anti-viral immune responses in vitro. PLoS One 6, e27898 (2011).

38. Garofalo, R. et al. Respiratory syncytial virus infection of human respiratory epithelial cells up-regulates class I MHC expression through the induction of IFN-beta and IL-1 alpha. J. Immunol. 157, 2506-2513 (1996).

39. Chu, H.W. et al. SPLUNC1 regulation in airway epithelial cells: role of Tolllike receptor 2 signaling. Respir. Res. 11, 155 (2010).

40. Maretzky, T. et al. ADAM10 mediates E-cadherin shedding and regulates epithelial cell-cell adhesion, migration, and beta-catenin translocation. Proc. Natl. Acad. Sci. USA 102, 9182-9187 (2005).

41. Primakoff, P. \& Myles, D.G. The ADAM gene family: surface proteins with adhesion and protease activity. Trends Genet. 16, 83-87 (2000).

42. Dijkstra, A. etal. Expression of ADAMs ("a disintegrin and metalloprotease") in the human lung. Virchows Arch. 454, 441-449 (2009).

43. Edwards, S.T. et al. c-Kit immunophenotyping and metalloproteinase expression profiles of mast cells in interstitial lung diseases. J. Pathol. 206, 279-290 (2005).

44. Rocks, N. et al. Expression of a disintegrin and metalloprotease (ADAM and ADAMTS) enzymes in human non-small-cell lung carcinomas (NSCLC). Br. J. Cancer 94, 724-730 (2006).

45. Wilke, G.A. \& Bubeck Wardenburg, J. Role of a disintegrin and metalloprotease 10 in Staphylococcus aureus alpha-hemolysin-mediated cellular injury. Proc. Natl. Acad. Sci. USA 107, 13473-13478 (2010).

46. Klein, E.Y., Sun, L., Smith, D.L. \& Laxminarayan, R. The changing epidemiology of methicillin-resistant Staphylococcus aureus in the United States: a national observational study. Am. J. Epidemiol. 177, 666-674 (2013).

47. Cao, J., Wong, C.K., Yin, Y. \& Lam, C.W. Activation of human bronchial epithelial cells by inflammatory cytokines IL-27 and TNF-alpha: implications for immunopathophysiology of airway inflammation. J. Cell. Physiol. 223, 788-797 (2010).

48. Reibman, J., Hsu, Y., Chen, L.C., Bleck, B. \& Gordon, T. Airway epithelial cells release MIP-3alpha/CCL2O in response to cytokines and ambient particulate matter. Am. J. Respir. Cell. Mol. Biol. 28, 648-654 (2003).

49. Paplinska, M., Chazan, R. \& Grubek-Jaworska, H. Effect of phoshpodiesterase 4 (PDE4) inhibibtors on eotaxin expression in humen bronchial epithelial cells. J. Physiol. Pharmacol. 62, 303-311 (2011).

50. Subauste, M.C., Choi, D.C. \& Proud, D. Transient exposure of human bronchial epithelial cells to cytokines leads to persistent increased expression of ICAM-1. Inflammation 25, 373-380 (2001).

51. Carayol, N. et al. Regulation of E-cadherin expression by dexamethasone and tumour necrosis factor-alpha in nasal epithelium. Eur. Respir. J. 20, 1430-1436 (2002)

52. Adson, A. et al. Quantitative approaches to delineate paracellular diffusion in cultured epithelial cell monolayers. J. Pharm. Sci. 83, 1529-1536 (1994). 